\title{
Simulations of Defect-Interface Interactions in GaN
}

\section{J. A. Chisholm and P. D. Bristowe}

Department of Materials Science and Metallurgy

University of Cambridge

Pembroke Street

Cambridge CB2 3QZ

United Kingdom

\section{ABSTRACT}

We report on the interaction of native point defects with commonly observed planar defects in GaN. Using a pair potential model we find a positive binding energy for all native defects to the three boundary structures investigated indicating a preference for native defects to form in these interfaces. The binding energy is highest for the $\mathrm{Ga}$ interstitial and lowest for vacancies. Interstitials, which are not thought to occur in significant concentrations in bulk GaN, should form in the (11 $\overline{2} 0)$ IDB and the $(10 \overline{1} 0)$ SMB and consequently alter the electronic structure of these boundaries.

\section{INTRODUCTION}

Vacancy and interstitial native defects are known to have a major influence on the electrical and optical properties of GaN [1]. For example, the N vacancy acts as single donor, the Ga vacancy as an acceptor and the interstitials act as amphoteric defects. The formation energies of native defects in bulk $\mathrm{GaN}$ have been calculated using first principles methods and their values are quite well established [2,3]. However, these formation energies will change in the neighbourhood of extended planar defects such as stacking mismatch boundaries and inversion domain boundaries. These boundaries are commonly observed in epitaxially grown $\mathrm{GaN}$ but so far the interaction between such interfaces and native point defects in $\mathrm{GaN}$ has not been investigated and it remains unclear whether the formation of interstitials and vacancies near the boundaries is encouraged or discouraged.

The calculation of defect-interface interactions requires computational cells containing several hundred atoms so as to avoid unwanted intercellular interactions when periodic boundary conditions are applied. This makes a first principles approach difficult and therefore we have used, in the first instance, a classical methodology which employs interatomic pair potentials that have been fitted to reproduce various bulk properties of $\mathrm{GaN}$. This classical model is used to calculate the binding energy of $\mathrm{Ga}$ and $\mathrm{N}$ vacancies and interstitials to three commonly observed interfaces: the (10 10) stacking mismatch boundary (SMB), the $(10 \overline{1} 0)$ inversion domain boundary (IDB) and the $(11 \overline{2} 0)$ IDB. The atomic structures of these boundaries have been determined from transmission electron microscope observations [4-6] and are shown in figure 1. One particular point of interest has been the observation of two atomic structures for the (10 $\overline{1} 0)$ IDB [7]. The first boundary structure involves an inversion of the atomic species across the boundary. For the second boundary structure, referred to as IDB*, there is an additional translation of $\mathrm{c} / 2$ along the [0001]. First principles density functional calculations carried out by 
Northrup at al [8] have shown that this second structure, which contains no dangling bonds, has the lower formation energy and we therefore focus on this structure in the present work. Analysis of the electronic structure also revealed that IDB* does not introduce any interface states in the forbidden gap but that the (10 $\overline{10})$ SMB introduces an occupied state $1.1 \mathrm{eV}$ above the valence band maximum.

a)

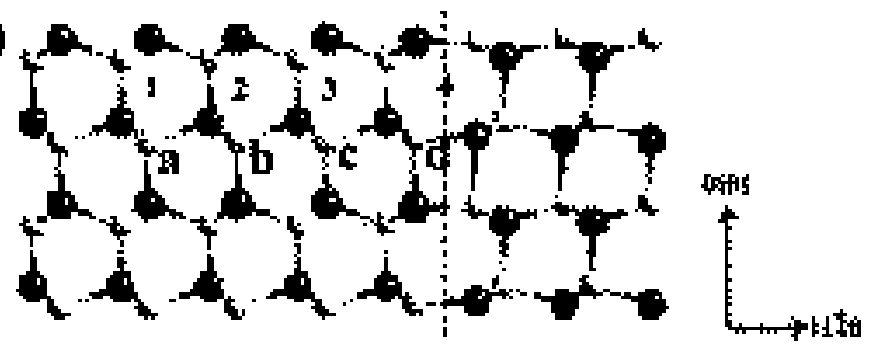

\$)

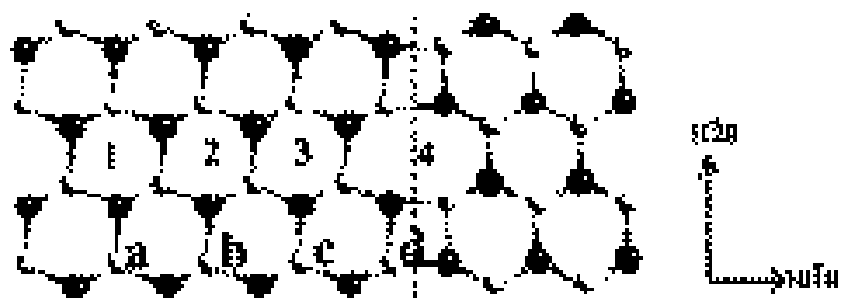

e)

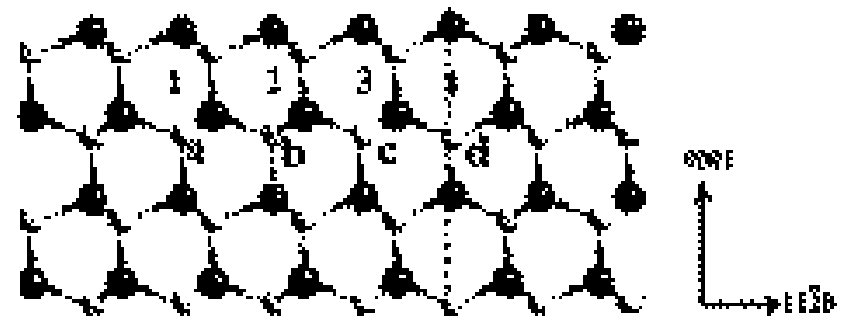

Figure 1. Observed boundary structures of (a) the (10 $\overline{1} 0) \mathrm{IDB}^{*}$, (b) the $(11 \overline{2} 0)$ IDB and (c) the $(10 \overline{1} 0)$ SMB. Interstitial defects are placed in positions 1-4 and vacancy defects are placed in positions a-d. Dashed line indicates the location of the boundary. 
The computational methodology and pair potential scheme are well established (for a review see Harding [9]) and therefore only a summary is given here. The long range electrostatic energy is evaluated through the Ewald summation [10]. The interaction between ions at short range is described using Buckingham pair potentials which have the following form:

$$
V_{i j}=A \exp \left(-r_{i j} / \rho\right)-C r_{i j}{ }^{-6}
$$

In a previous study [11] the potential parameters $A, \rho$ and $C$ were fitted to experimental lattice parameters as well as elastic and dielectric constants. For the $\mathrm{N}$ ion, polarisation effects are taken into account using the Shell Model in which the electrons are modelled by a massless 'shell' with charge $Y$ which is connected to the core by a spring with spring constant $K[12]$. The polarisability is then given by $\alpha=Y^{2} / K$.

The use of the supercell approach requires the inclusion of two boundaries per cell. We have carried out convergence tests and found that the following sizes of supercell are more than adequate to eliminate boundary-boundary interactions as well as interactions between native defects: $(10 \overline{1} 0)$ IDB* 576 atoms, $(11 \overline{2} 0)$ IDB 528 atoms and (10 $\overline{1} 0)$ SMB 416 atoms. For each calculation we allow full relaxation of the cell parameters and of the atomic coordinates by using a Broyden-Fletcher-Goldfarb-Shanno scheme. Our approach for calculating native defect binding energies is shown in figure 1 . The binding energy is the difference between the formation energy of the defect in the bulk and the formation energy of the defect in the boundary. This change in formation energy is obtained simply from the change in the total energy of the supercell as the native defect is taken from the bulk environment and placed in the boundary.

$$
\mathrm{E}_{\text {bind }}=\mathrm{E}_{\mathrm{tot}}(\text { defect in bulk })-\mathrm{E}_{\mathrm{tot}}(\text { defect in boundary })
$$

In the pair potential model the atoms are treated as ions in which the $\mathrm{Ga}$ atom is attributed a charge of +2 and the $\mathrm{N}$ atom a charge -2 . The creation of a native defect therefore introduces an overall charge into the supercell. In order to maintain charge neutrality we create an additional native defect. For example, for a $\mathrm{N}$ vacancy we also create a $\mathrm{Ga}$ vacancy the position of which is kept fixed at the largest possible distance from the $\mathrm{N}$ vacancy. Again, the size of the supercells in each case is more than sufficient to eliminate any unwanted defect-defect interactions.

\section{RESULTS}

Calculated values for the boundary energies as well as native defect binding energies are shown in table I. The boundary energies compare well with those obtained by Northrup et al [8] who calculated values of $0.025 \mathrm{eV} / \AA^{2}$ for the $(10 \overline{10})$ IDB* and 0.105 $\mathrm{eV} / \AA^{2}$ for the $(10 \overline{10}) \mathrm{SMB}$.

Trends for the binding energies can be observed across the boundaries and across the native defects. We note first that for all boundary structures the binding energy is higher 
for interstitial defects than for vacancies. In bulk GaN, the interstitials have a particularly high formation energy of 5-10 eV (depending on the charge state [1]) and unlike the case for GaAs, interstitials are not thought to occur in significant concentrations. This result suggests that interstitial defects are more likely to form in the boundary. Indeed, a larger volume of space is available to accommodate interstitials for all three boundaries.

Table I. Calculated binding energies for $\mathrm{Ga}$ and $\mathrm{N}$ vacancies and interstitials to three different planar defects. The boundary energy of each planar defect is also given.

\begin{tabular}{|c|c|c|c|}
\hline & $(10 \overline{1} 0) \mathrm{IDB}^{*}$ & $(11 \overline{2} 0) \mathrm{IDB}$ & $(10 \overline{1} 0) \mathrm{SMB}$ \\
\hline $\begin{array}{c}\text { Boundary energy } \\
\left(\mathrm{eV} / \AA^{2}\right)\end{array}$ & 0.017 & 0.215 & 0.095 \\
\hline $\mathrm{Ga}$ interstitial $(\mathrm{eV})$ & 1.46 & 5.31 & 5.28 \\
\hline $\mathrm{N}$ interstitial $(\mathrm{eV})$ & 1.04 & 3.95 & 4.40 \\
\hline $\mathrm{Ga}$ vacancy $(\mathrm{eV})$ & 0.44 & 1.61 & 2.39 \\
\hline $\mathrm{N}$ vacancy $(\mathrm{eV})$ & 0.43 & 1.90 & 2.79 \\
\hline
\end{tabular}

In bulk $\mathrm{GaN}$ the introduction of a Ga interstitial causes an outward relaxation of the surrounding atoms. The same is generally true for an interstitial introduced into the boundaries. For the case of the $(10 \overline{10}) \mathrm{IDB}^{*}$, one particular Ga atom in the boundary core is displaced outward by $1.0 \AA$ because of the attraction of the Ga interstitial to three nearby $\mathrm{N}$ ions. This is shown in figure 2 (a). For the $(11 \overline{2} 0)$ IDB, two $\mathrm{N}$ atoms from the boundary are displaced inwards towards the Ga interstitial by $0.7 \AA$ and $0.6 \AA$ and a Ga atom in the boundary is pushed out by $0.8 \AA$. Also, for the (10 $\overline{1} 0) \mathrm{SMB}$, there is a small rearrangement whereby surrounding $\mathrm{Ga}$ atoms relax outwards. For this boundary there are no inward relaxations of surrounding $\mathrm{N}$ atoms due to the presence of $\mathrm{N}$ atoms located directly in the boundary core. A similar result holds for the $\mathrm{N}$ interstitial only that now surrounding $\mathrm{Ga}$ atoms relax inwards to meet the $\mathrm{N}$ interstitial and $\mathrm{N}$ ions are pushed out as shown in figure 2 (b) for the case of the $(10 \overline{1} 0)$ IDB*.

A careful comparison of lattice relaxations carried out within a radius of $7 \AA$ for all native defects has shown that displacements are of the same magnitude both in the boundary and in the bulk. Also, it was seen that displacements associated with vacancies are less pronounced than those associated with interstitials. For the Ga vacancy in the (10 10$)$ IDB*, two $N$ ions relax outward by $0.4 \AA$ and $0.2 \AA$ and a Ga ion moves towards the vacancy by $0.3 \AA$. For the $(11 \overline{2} 0)$ IDB, N ions are observed to move out by $0.5 \AA$ and $0.2 \AA$ and there are significant displacements for two nearby Ga ions: $0.6 \AA$ 
and $0.8 \AA$. For the SMB, where a Ga ion is only three-fold coordinated, surrounding N ions relax outward by $0.2,0.2$ and $0.1 \AA$. Again the situation is similar for the $\mathrm{N}$ vacancy only now $\mathrm{N}$ ions are observed to move a fraction of an Ångstrom in towards the $\mathrm{N}$ vacancy and surrounding Ga ions relax outwards.

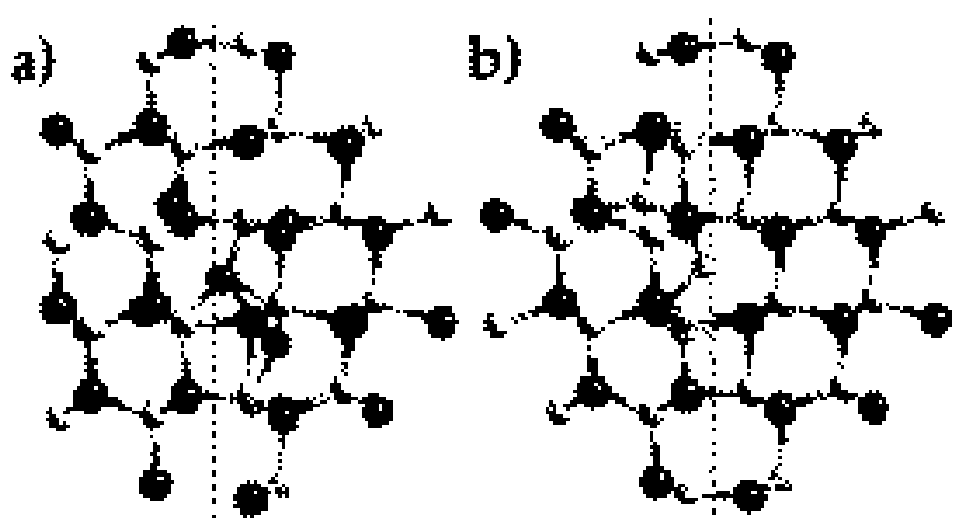

Figure 2. Relaxed structures of (a) the Ga interstitial and (b) the $\mathrm{N}$ interstitial in the $\left(10 \overline{1}^{0}\right) \mathrm{IDB}^{*}$. Filled and open circles represent gallium and nitrogen atoms respectively. Dashed line indicates the location of the boundary.

The electronic structure of a boundary will be affected by the formation of native defects in its core. In the case of the $(10 \overline{1} 0)$ IDB $^{*}$, the formation of vacancies and interstitials will cause the boundary to become electrically active. The concentration of such native defects in the boundary will depend on the point defect formation energy in the boundary environment. Defect-interface binding energies shown in table I indicate that the formation energies of interstitials are not significantly less than bulk values for this particular boundary. However, formation energies are significantly reduced for interstitials in the $(11 \overline{2} 0)$ IDB and the $(10 \overline{10})$ SMB. The precise nature of such defects in the boundary requires a first principles approach and such a treatment is currently underway.

We note also that the binding energies follow a definite trend across the boundaries. For the case of the interstitials this can be explained in terms of the volume which is available to accommodate the defect. An interstitial can be thought of as being enclosed in a cage of atoms having a certain volume. Analysis shows that the volume of these interstitial cages increases in going from the $(10 \overline{10}) \mathrm{IDB}^{*}$ to the $(11 \overline{2} 0)$ IDB to the (10 10$)$ SMB which correlates well with the corresponding increase in binding energies for the native defects across this series. The apparent smaller cage size of the SMB in figure 1 is due to the orientation of the figure. 


\section{CONCLUSIONS}

We have employed a pair potential model to investigate the interaction between native defects and inversion domain boundaries and stacking mismatch boundaries in $\mathrm{GaN}$. The binding energy is found to be positive for all the native defects and boundary structures considered. This indicates that native defects prefer to form in these types of boundary. The binding energy is highest for the Ga interstitial and lowest for the vacancies which can be explained by the larger volume available to accommodate interstitials in the boundary. We conclude that $\mathrm{N}$ and $\mathrm{Ga}$ interstitials, which do not form in significant concentrations in bulk GaN, should form in the (11 $\overline{2} 0)$ IDB and the (10 $\overline{1} 0)$ SMB. Investigations using first principles calculations are currently underway to confirm the trends in binding energies found in this study as well as gain information on the electronic structure of these boundaries in the presence of native defects.

\section{ACKNOWLEDGEMENT}

Support for this research was provided by the Engineering and Physical Sciences Research Council.

\section{REFERENCES}

1. J. Neugebauer and C. G. Van de Walle, Phys. Rev. B 50, 8067 (1994).

2. P. Boguslawski, E. L. Briggs and J. Bernholc, Phys. Rev. B 51, 17255 (1995).

3. J. Neugebauer and C. Van de Walle, Festkorperprobleme-Advances in Solid State Physics, 35, 25 (1996)

4. L. T. Romano, J. E. Northrup and M. A. O’Keefe, Appl. Phys. Lett., 69, (16), 2395 (1996).

5. Y. Xin, P. D. Brown, C. J. Humphreys, T. S. Cheng and C. T. Foxon, Appl. Phys. Lett., 70, (10), 1308 (1997).

6. S. Ruvimov, Z. Liliental-Weber, J. Washburn, H. Amano, I. Akasaki, and M. Koike, MRS Symposium Proceedings, 482, 387 (1998)

7. V. Pontin, G. Nouet and P. Ruterana, Appl. Phys. Lett., 74, 947 (1999).

8. J. E. Northrup, J. Neugebauer and L. T. Romano, Phys. Rev. Lett., 77, 103 (1996).

9. J. H. Harding, Rep. Prog. Phys., 53, 1403 (1990).

10. P. P. Ewald, Ann. Phys., 64, 253 (1921).

11. J. A. Chisholm, D. W. Lewis and P. D. Bristowe, J. Phys. Condensed Mat., 11, L235 (1999).

12. B. G. Dick and A. W. Overhauser, Phys. Rev., 112, 90 (1958). 